Musées, Patrimoine et Culture scientifiques et techniques

$153 \mid 2014$

mai-juin 2014

\title{
La diffusion des collections liée au récolement
}

Nathalie Mémoire pour la Conférence Permanente des Muséums de France

\section{OpenEdition}

Journals

Édition électronique

URL : http://journals.openedition.org/ocim/1410

DOI : $10.4000 /$ ocim. 1410

ISSN : 2108-646X

Éditeur

OCIM

Édition imprimée

Date de publication : 25 juin 2014

ISSN : 0994-1908

\section{Référence électronique}

Nathalie Mémoire pour la Conférence Permanente des Muséums de France, «La diffusion des

collections liée au récolement », La Lettre de l'OCIM [En ligne], 153 | 2014, mis en ligne le 25 juin 2016, consulté le 01 mai 2019. URL : http://journals.openedition.org/ocim/1410 ; DOI : 10.4000/ocim.1410 


\title{
La diffusion des collections liée au récolement
}

\author{
Nathalie Mémoire \\ pour la Conférence Permanente des Muséums de France
}

La journée consacrée au récolement organisée en décembre 2013 par le Service des Musées de France axait la réflexion autour de deux thèmes : récolement et nouvelles réserves et récolement et diffusion. Tandis que le muséum de Nantes était invité à intervenir sur la question des réserves mutualisées, la Conférence Permanente des Muséums de France (CPMF) s'interrogeait sur la thématique de la diffusion.

Comme il a été dit, le chantier des collections dans les muséums n'a pas commencé avec l'obligation du récolement mais a été entrepris dès la décennie 1990 (et même 1981 à Grenoble). En effet, pour la plupart d'entre eux, c'est la possibilité de créer des bases de données informatisées qui a motivé la réalisation d'inventaires documentaires. Pour certains, comme à La Rochelle, Lyon, ou Bordeaux, c'est le projet de rénovation complète de l'établissement qui a conduit à l'intensification de ces chantiers, pour d'autres, c'est le réaménagement de nouvelles salles ou la création de nouvelles réserves (Nancy, Saint-Quentin).

Lobligation du récolement a créé une dynamique nationale qui a souvent permis de sensibiliser les élus et d'accélérer la réalisation des chantiers (Bayonne, Lyon...) grâce à l'obtention de moyens supplémentaires (personnel occasionnel, locaux), et l'implication des équipes. Mené sur plusieurs années, il a considérablement amélioré le niveau de documentation des collections (notamment pour l'origine des biens, la couverture photographique, les constats d'état) et, en conséquence, la connaissance globale des fonds de collections. L'enrichissement des bases de données des collections qu'il a engendré, a conforté les perspectives de valorisation des collections qui se traduisent déjà ou devraient se traduire à terme par :

- la consultation en ligne des collections par le public ; - le versement des données dans les bases et portails nationaux et internationaux.

Ainsi, Le Mans et Strasbourg, mais aussi Nice, Nancy, Angers, Aix-en Provence, Auxerre, Bourges, Montbéliard, Rouen, entre autres, ont-ils déjà alimenté certaines de ces bases spécialisées, en particulier : Trans' Tyfipal ${ }^{\circledR}$, GBIF, Tela Botanica (réseau collaboratif de botanistes francophones), Sonnerat... (voir l'article de Marie-Dominique Wandhammer et Nicolas Morel, p. 79 et le texte de Jérôme Thomas, p. 84).

Des publications ont pu directement bénéficier de la documentation des collections, comme la collection sur le Patrimoine géologique (synthèses sur chacun des stratotypes, sites de référence internationale situés en France) à laquelle ont contribué entre autres les muséums du Mans, d'Aix-en-Provence, de Bordeaux. Des articles concernant directement les problématiques de conservation ont également été publiés dans plusieurs revues spécialisées (Rouen). Renforçant la connaissance historique des collections, notamment les liens transversaux entre objets appartenant à des domaines différents, le récolement devrait permettre de publier un certain nombre de catalogues raisonnés - Montbéliard-muséum Cuvier (voir l'article de Thierry Malvesy, p. 86), Clermont-Ferrand - et aussi d'envisager de nouveaux sujets d'études sur les collections.

D'autre part, le récolement conduisant à mieux connaître et documenter les collections nous permet de planifier une meilleure valorisation via le réaménagement de nouveaux espaces (Montbéliard-muséum Cuvier, Nancy, Auxerre) et les programmations d'expositions à venir.

Des expositions spécifiques présentant au public les chantiers des collections ont été réalisées à Auxerre, Lyon, Châteaudun, Bordeaux, et d'autres sont prévues en 2014 (Aix-en-Provence, Montbéliard-muséum Cuvier, Bordeaux, Nancy), ainsi que de nombreuses visites des réserves et des chantiers lors des Journées européennes du Patrimoine. La presse s'est souvent fait l'écho de ces manifestations.

La découverte ou redécouverte de spécimens rares qui serait due au récolement reste exceptionnelle (Toulon, Auxerre) ou anecdotique. En revanche, la mise en ligne de nos bases de données devrait fortement accroître le nombre de demandes de consultations et d'études.

En conclusion, les muséums dans leur ensemble se reconnaissent davantage dans une synergie de projets plus que dans l'application directe d'une obligation règlementaire. Il est néanmoins incontestable que le récolement comme objectif partagé a permis de sensibiliser les élus, accélérer des projets, motiver les équipes et informer le public d'une part de l'activité restée jusqu'alors peu visible. 


\section{Le récolement?... Ca colle ! 10 ans de récolement des collections}

Lors de la réunion de la Conférence Permanente des Muséums de France du 27 septembre 2013, Catherine Gautier (directrice du muséum de Grenoble) suggérait que nous utilisions notre réseau pour créer de manière collaborative des contenus d'expositions qui pourraient être déclinés et enrichis par chacun selon ses espaces, ses moyens et ses spécificités de collections comme de public.

Une thématique déjà largement travaillée en commun au cours des deux dernières années pouvait être celle du récolement, une exposition sur le sujet lui-même permettant d'expliquer à un large public comme aux élus les enjeux nationaux mais aussi les spécificités partagées de nos muséums, le potentiel des collections et le travail des acteurs. Concrètement, la préparation d'articles pour La Lettre de l'OCIM sur le sujet pouvait être la source documentaire servant de base aux textes de l'exposition. Des photographies représentatives, témoignant du travail dans différents musées, pouvaient aisément venir illustrer le propos.

L'exposition collaborative se décline en une douzaine de panneaux formant un tronc commun. Les premiers décrivent les obligations, les enjeux et les échéances pour l'ensemble des « Musées de France ». Sont ensuite évoquées les problématiques partagées au sein de la CPMF, les stratégies mises en œuvre par chacun, enfin la valorisation des collections qui peut déjà et pourra être faite suite à ces chantiers. En complément, un nombre variable de panneaux, zoomant sur chaque établissement, permettra de développer les particularités de chacun en termes d'histoire, de volume de collections, de méthodologie et moyens mis en œuvre, enfin de perspectives et de suites à donner.

Ainsi, une première déclinaison sera présentée à Bordeaux du 13 juin au 21 septembre 2014. À la partie commune s'ajouteront six panneaux sur le muséum de Bordeaux et un sur le contexte des musées en Aquitaine, ainsi qu'un large échantillon des collections, représentatif de leur diversité, permettant d'éclairer concrètement le propos.
Les panneaux (ou seulement les textes dans une autre mise en page adaptée aux chartes des établissements) seront utilisés, dès juillet à Aix-en-Provence pour une exposition sur le chantier des collections, pour les Journées européennes du Patrimoine à Nantes, Bourges, Lille et Saint-Quentin en Picardie qui poursuivra avec la Fête de la Science, cette occasion étant également celle choisie par Angers ; Clermont-Ferrand utilisera les contenus sous forme d'un diaporama, Nancy utilisera quelques extraits des textes dans une exposition centrée sur le déménagement de ses collections, Rouen, Grenoble et Nice sous une forme encore à préciser.

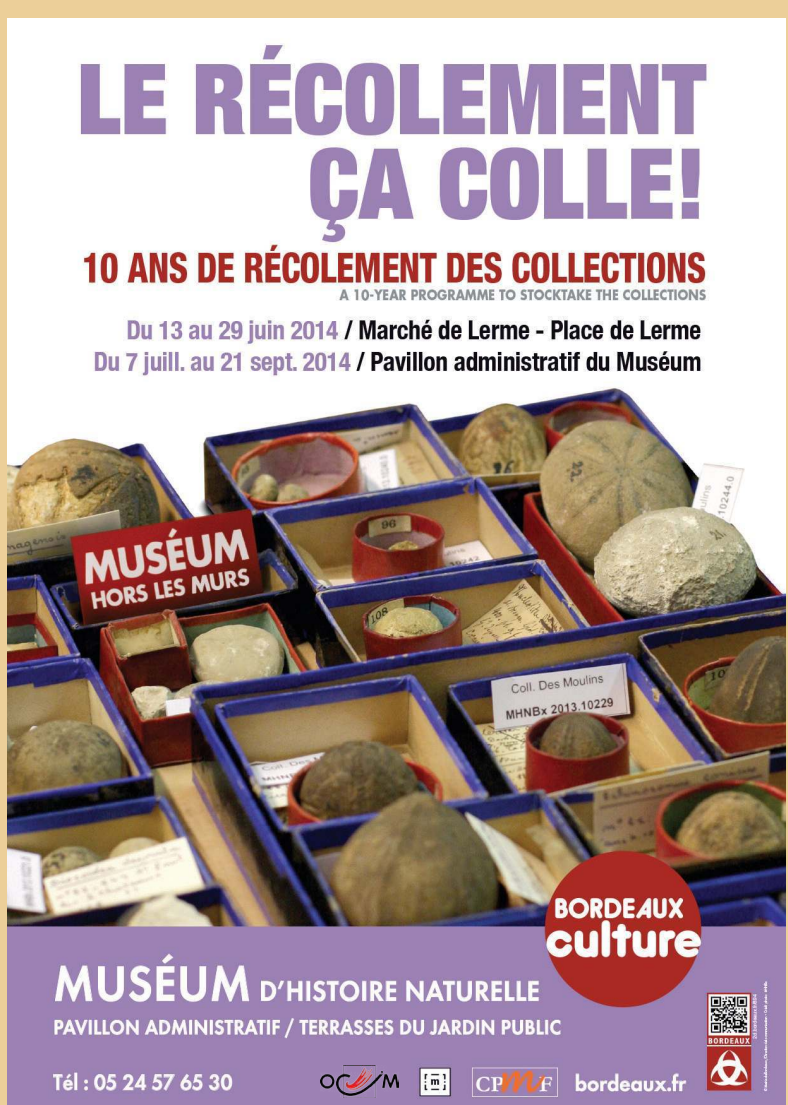

